\title{
Toma de decisiones en la retirada del tratamiento renal sustitutivo: aspectos éticos
}

\section{Ethical issues on the decision-making process towards dialysis withdrawal}

\author{
Alejandro Vega-Hernández ${ }^{1 *}$ y Janet Delgado-Rodríguez $z^{2,3}$ \\ ${ }^{1}$ Subdirección de Enfermería, Complejo Hospitalario Universitario Insular Materno-Infantil, Las Palmas, Las Palmas de Gran \\ Canaria; '2Subdirección de Enfermería, Hospital Universitario de Canarias, Santa Cruz de Tenerife, La Cuesta; ${ }^{3}$ Instituto \\ Universitario de Estudios de las Mujeres, Universidad de La Laguna, Santa Cruz de Tenerife, San Cristóbal de La Laguna. \\ España
}

\section{Resumen}

El proceso de toma de decisiones acerca de la terapia renal sustitutiva es complejo y depende de muchos factores, tanto al elegir la modalidad como al optar por un manejo conservador. Cuando se trata de adecuar el esfuerzo terapéutico, la retirada de la terapia renal sustitutiva (RTRS) supone todo un desafío ético para los profesionales, los pacientes y sus familiares. El objetivo principal de este artículo es identificar y analizar las recomendaciones éticas disponibles respecto a la RTRS. Para ello, se llevó a cabo una revisión de la literatura en PubMed, Cinahl, Cochrane, Lilacs y Medes, y se seleccionaron 55 artículos. Los resultados fueron organizados en siete áreas. Cuando se plantea la RTRS, los desafíos a la autonomía del paciente son constantes, por lo que la toma de decisiones compartida puede ser un instrumento para minimizar la vulneración de este principio. La RTRS se debe realizar con medidas adecuadas y acompañarse de soporte paliativo. Se necesita una mayor formación de los profesionales sanitarios en habilidades comunicativas y bioética.

Palabras clave: Bioética. Hemodiálisis. Diálisis. Nefrología. Retirada.

\begin{abstract}
The decision-making process about RRT is complex and depends on many factors, whether modality of RRT or the option of conservative management are chosen. When it comes to tailoring therapeutic effort, dialysis withdrawal (DW) poses a challenge for professionals, patients and relatives. The aim of this paper is to identify and analyze ethical recommendations about DW. PubMed, Cinahl, Cochrane, Lilacs and Medes databases were searched and 55 articles chosen. The results were organized in 7 areas. When discussing DW, challenges towards patient's autonomy are constant, shared decision-making could be an instrument to minimize

\section{Correspondencia:}

*Alejandro Vega-Hernández

E-mail: vegahernandezalejandro@gmail.com

2395-938X / @ 2021 Centros Culturales de México, A.C. Publicado por Permanyer. 2395-938X / @ 2021 Centros Culturales de México, A.C. Publicado por Permanyer. Éste es un artículo open access bajo la licencia
CC BY-NC-ND (http://creativecommons.org/licenses/by-nc-nd/4.0/).

Fecha de recepción: 12-12-2020

Fecha de aceptación: 30-03-2021

DOI: $10.24875 /$ BUP.20000003

Disponible en internet: 21-05-2021 BIOETHICS UPdate 2021;7(1):35-50 www.bioethicsupdate.com
\end{abstract}


violations of this principle. DW must be done in a proper way and supported with palliative care. Professionals need more formation in communication abilities and bioethics.

Key words: Bioethics. Haemodialysis. Renal dialysis. Nephrology. Withdrawal.

\section{Introducción}

En la actualidad, la enfermedad renal crónica (ERC) supone un grave desafío para los sistemas de salud. Por un lado, esta enfermedad va acompañada de un incremento en la morbilidad y complica cualquier otra patología pudiendo, por ejemplo, ser un factor de riesgo cardiovascular independiente ${ }^{1}$. Por otro lado, su coste económico es muy elevado: en España solo la terapia renal sustitutiva (TRS) consume el $2.5-3 \%$ del presupuesto del Sistema Nacional de Salud. El coste medio anual por paciente en diálisis es 6 veces mayor que el de pacientes con infección por VIH y 24 veces mayor que el de pacientes con enfermedad pulmonar obstructiva crónica y asma ${ }^{1}$. La ERC es tratable y potencialmente prevenible, lo que añade gran importancia a su detección precoz.

La decisión sobre el tratamiento a seguir se toma conjuntamente con el paciente, una vez que ha sido informado adecuadamente del pronóstico y de cada modalidad de TRS. La Sociedad Española de Nefrología ${ }^{1}$ nos recuerda que: «El tratamiento conservador también debe ser considerado y ofrecido a aquellos pacientes en los que sus circunstancias clínicas lo aconsejen [...] es importante que el paciente esté informado de todas las opciones y conozca que la transferencia entre las mismas es posible $y$, a veces, beneficiosa»1.

Cuando un paciente que ya está en TRS es susceptible de pasar a tratamiento conservador, nos encontramos ante la denominada retirada del tratamiento renal sustitutivo (RTRS). Esta decisión puede provenir del médico o del paciente y estar motivada por cambios de la situación clínica o personal en el tiempo. La RTRS es un proceso complejo con numerosos desafíos, puesto que se detiene un tratamiento de soporte vital que supondrá la muerte del paciente en un corto plazo². Para lograr un acuerdo satisfactorio deben equilibrarse los valores y creencias del paciente, de sus familiares y de los profesionales ${ }^{3}$, y en este proceso pueden verse comprometidos todos los principios bioéticos clásicos: autonomía, beneficencia, no maleficencia y justicia.

Son numerosas las dificultades para la investigación de la RTRS. Se trata de un campo disperso que dificulta las estrategias de búsqueda ${ }^{4,5}$, los artículos presentan escasa evidencia o son inconsistentes ${ }^{6,7}$ y falta un criterio unificado de clasificación ${ }^{8}$, lo que afecta directamente a sus estadísticas. Por ejemplo, Qazi, et al. ${ }^{6}$ señalan que, de 15 estudios analizados, solo 3 usaban una definición específica de RTRS, mientras el resto la consideraban como cualquier discontinuación de la técnica. Muchos autores no reflejan si la decisión de RTRS la toma el médico o el paciente ni los factores éticos involucrados ${ }^{9}$. Además, existe una importante variación entre las prácticas de distintos países e incluso entre centros del mismo país ${ }^{10,11}$.

Las principales asociaciones nefrológicas han dado algunas indicaciones para ayudar a los profesionales a manejar situaciones de RTRS. Sin embargo, estas recomendaciones no recogen de manera amplia los problemas éticos implicados, un tema que tanto profesionales como 
pacientes consideran muy relevante ${ }^{3,6,11-13}$. A pesar de que la RTRS es una forma de adecuación del esfuerzo terapéutico (AET), persisten los miedos y confusiones en torno a ella.

El objetivo principal de este artículo es identificar y analizar las recomendaciones éticas publicadas actualmente respecto a la RTRS. Además, se persigue identificar áreas donde pudieran ser de utilidad nuevas recomendaciones bioéticas.

\section{Metodología}

\section{Estrategia de búsqueda}

En la tabla 1 se describe la estrategia de búsqueda. Se emplearon varios términos usando los tesauros de la DeCS (descriptores en Ciencias de la Salud) y el MeSH (Medical Subject Headings) para comprobar la validez de los descriptores, tanto en español como en inglés. La búsqueda bibliográfica se realizó en febrero de 2020 en las bases de datos PubMed, Cinahl, Cochrane, Lilacs y Medes. La selección de los estudios se realizó a partir de los títulos y resúmenes recuperados en las bases de datos aplicando los criterios de inclusión y exclusión. Los artículos seleccionados fueron analizados a texto completo. Se incluyeron estudios experimentales y cuasiexperimentales, cualitativos, observacionales, revisiones sistemáticas y narrativas y análisis de casos. Se excluyeron cartas al editor y resúmenes de congresos.

\section{Resultados}

Tras aplicar la estrategia de búsqueda en las diversas bases de datos (Fig. 1), fueron seleccionados 54 artículos. Se añadió manualmente un libro por su relevancia para el tema. Los artículos procedían de EE.UU., Reino Unido, España, Chile, Cuba, Colombia, Brasil, Alemania, Suecia, Sudáfrica, Australia, Japón y Taiwán. El tipo de artículos fueron: revisión narrativa (18), estudios de casos (11), revisiones sistemáticas (6), estudios cualitativos (3), estudios cuantitativos mediante encuesta (3), estudio observacional y/o descriptivo (3), desarrollo de guías (2), argumentación ética (1), ensayo aleatorio (1), estudio de casos y controles (1), análisis estadístico de casos (1), libro (1) y otros (4). Para el análisis de los resultados se siguió la clasificación de Kahrass, et al. ${ }^{5}$. Se recoge una síntesis de los resultados en la tabla 2.

\section{Problemas éticos en el diagnóstico, pronóstico e indicaciones médicas}

Con ligeras variaciones entre países, el 40\% de las personas mayores de 75 años está diagnosticada de ERC, aunque puede haber sobrediagnóstico ${ }^{14}$. En España, el 21.4\% de los españoles > 65 años tienen ERC diagnosticada ${ }^{1}$. Diversos autores defienden que la pregunta ética debe cambiar de “¿quién debe recibir diálisis?" a “¿quién no debe recibir diálisis?»15,16. La edad cronológica no es un criterio de decisión válido para excluir pacientes de TRS, antes bien, se debe basar la decisión en la ponderación del riesgo-beneficio del tratamiento y las preferencias de los pacientes ${ }^{17}$. 
Tabla 1. Estrategia de búsqueda

\begin{tabular}{|l|l|}
$\begin{array}{l}\text { Descriptores } \\
\text { Español } \\
\text { Inglés }\end{array}$ & $\begin{array}{l}\text { Bioética, hemodiálisis, diálisis, nefrología, retirada } \\
\text { Bioethics, haemodialysis, renal dialysis, nephrology, withdrawal }\end{array}$ \\
\hline Operadores & AND \\
\hline Criterios de inclusión & $\begin{array}{l}\text { Alusión a aspectos éticos } \\
\text { Referencia a la retirada o no inclusión de la terapia renal sustitutiva } \\
\text { Idioma inglés o español }\end{array}$ \\
\hline Criterios de exclusión & $\begin{array}{l}\text { No aborda aspectos éticos } \\
\text { Idioma distinto al español o inglés } \\
\text { Relacionado con trasplantes o diálisis peritoneal } \\
\text { Artículos repetidos de otras búsquedas o bases de datos } \\
\text { Artículos inaccesibles de manera pública o por medio de la ULPGC }\end{array}$ \\
\hline
\end{tabular}

ULPGC: Universidad de Las Palmas de Gran Canaria.

No obstante, en pacientes mayores con múltiples comorbilidades, la diálisis no proporciona un incremento del tiempo de vida respecto al tratamiento conservador y, sin embargo, sí se asocia a un deterioro físico y funcional, un mayor riesgo de sufrir hospitalizaciones, una reducción general de la calidad de vida ${ }^{14,18-20}$ y una alta mortalidad, sobre todo en los primeros seis meses ${ }^{1,21,22}$.

Se estima que solo el $75 \%$ de los pacientes > 75 años que comienzan TRS vivirá más de un año ${ }^{23} \mathrm{y}$, además, se habla del «síndrome ERCA de residencia»: iniciar TRS en pacientes que viven en residencias se asocia a un rápido deterioro funcional y a una mortalidad significativa ${ }^{23}$. Aun así, son un grupo destacado en las nuevas admisiones a TRS. ¿Es correcto aplicar un tratamiento agresivo con limitadas expectativas de éxito al paciente? ${ }^{24}$. Cranston ${ }^{25}$ ya avisa de los riesgos de seguir el imperativo tecnológico (puedo, luego debo) pero también de los desafíos éticos de no hacerlo. Por otro lado, los pacientes en tratamiento conservador mantienen su nivel funcional durante el último año de vida hasta un deterioro agudo en el último mes, mientras que los pacientes en TRS experimentan ese deterioro mucho antes ${ }^{20}$. La incertidumbre en torno a la evolución de la enfermedad genera una gran barrera para el paciente y el profesional a la hora de decidir la $\mathrm{RTRS}^{26}$. Se retrasa la conversación sobre las expectativas de vida hasta agotar todas las alternativas $^{12,13}$ y ya se ha llegado a un deterioro importante o a la propia fase terminal.

\section{Problemas éticos en la diálisis}

El aumento de casos de RTRS va ligado al aumento de personas frágiles con comorbilidades admitidas en TRS ${ }^{27}$, sobre todo > 75 años ${ }^{11}$. Aumentan también los datos de mortalidad relacionada con RTRS ${ }^{8}$. Las conversaciones acerca de la RTRS pueden suponer un desafío emocional para todos los implicados ${ }^{26}$. La Renal Physicians Association recomienda los cuidados paliativos renales (CPR) para que todos los pacientes con $\mathrm{ERCA}^{11}$ se preparen con tiempo ${ }^{26}$, pero las creencias y valores de los profesionales tendrán un impacto en la integración de los cuidados paliativos en el paciente renal ${ }^{21}$. Además, el marco legal de actuación sigue siendo desconocido entre los profesionales. En España, el nefrólogo tiene la responsabilidad de ofrecer al paciente un tratamiento que considere beneficioso y no maleficente, pero no está obligado ni ética ni legalmente 


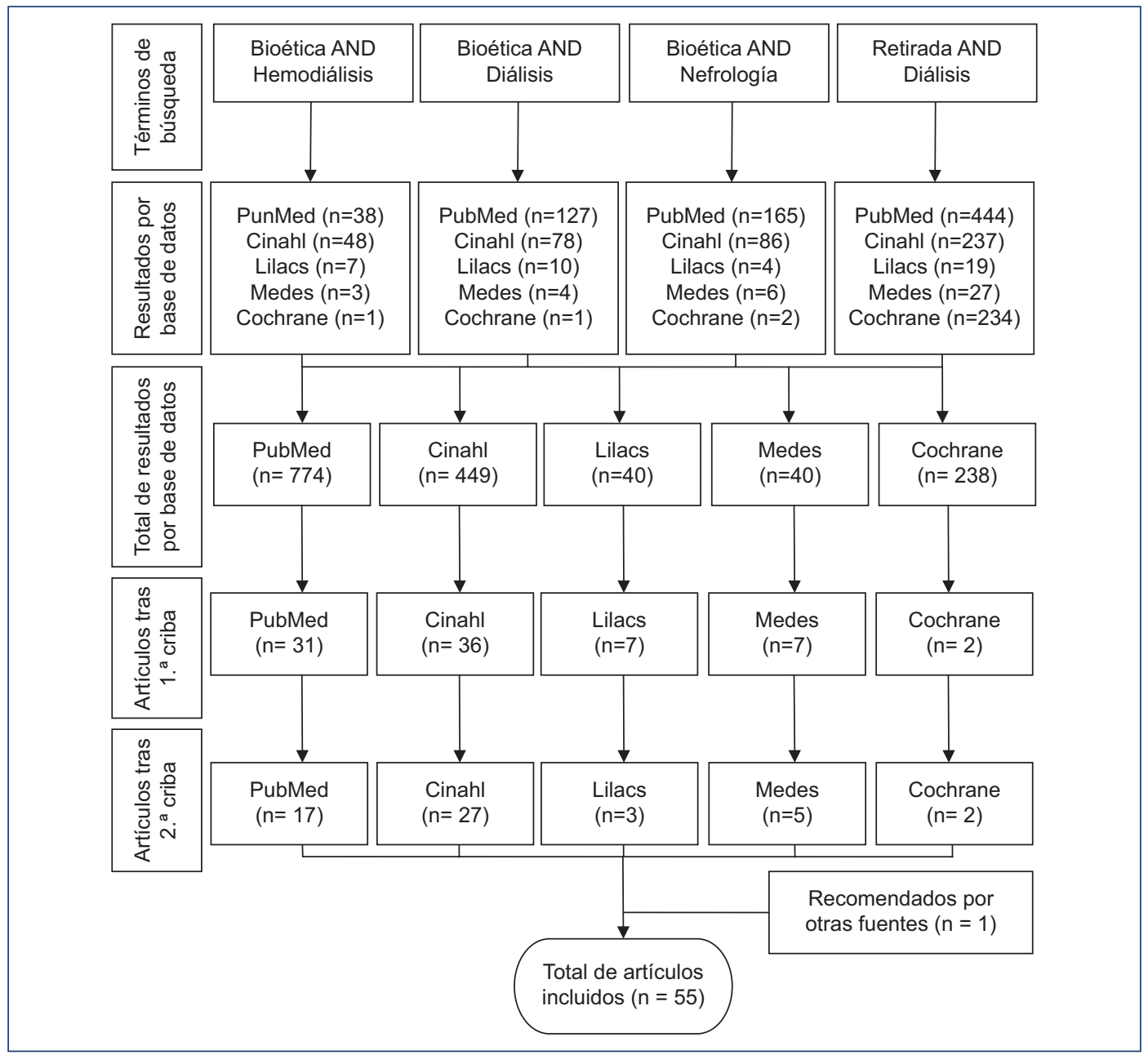

Figura 1. Selección de estudios.

a proporcionárselo si considera inapropiado ese tratamiento para él1. Por tanto, un médico no incurre en delito civil o penal por rechazar el iniciar o continuar la TRS de un paciente bajo su criterio profesional. No pasa lo mismo por comenzar o continuar el tratamiento a un paciente que no da su consentimiento; esto sí que puede ser considerado un crimen por vulneración del principio de autonomía ${ }^{28}$. En lo referente al principio de autonomía y la doctrina del doble efecto, no hay una diferencia ética respecto a retirar o no iniciar un tratamiento vital ${ }^{23}$, por lo que no puede ser considerado una forma de suicidio asistido ${ }^{28}$.

La hemodiálisis (HD) discontinua es extremadamente costosa en tiempo para el paciente. Aunque se prolongue la expectativa de vida, va a pasar una parte significativa de este tiempo en un entorno hospitalario ${ }^{4}$, con mayores tasas de hospitalizaciones, admisiones en $\mathrm{UCl}$ y tratamientos agresivos $^{14}$. El uso de TRS puede ser cuestionado si no se logra mejorar la calidad de vida en pacientes con escasas perspectivas de supervivencia ${ }^{2}$. Para estos pacientes (por su expectativa de vida corta $[<6$ meses], mala situación funcional [dependencia de las actividades de la vida 
Tabla 2. Principales hallazgos de la revisión clasificados según el modelo propuesto por Kahrass, et al., $2016^{5}$

Hallazgos principales

Artículos

Problemas éticos en el diagnóstico, pronóstico e indicaciones médicas

- Dificultades para la exclusión de pacientes de diálisis

- Dificultad al establecer un pronóstico y planificar al respecto

- Edad cronológica criterio inaceptable para decidir por sí solo

- Criterio basado en ventajas-inconvenientes y preferencias del paciente

- > 75 años pobre ganancia de tiempo de vida y peor deterioro funcional: síndrome ERCA de residencia

- La diálisis no se considera un tratamiento prima facie beneficioso para el paciente $>75$ años

- Buena calidad de vida del paciente que opta por tratamiento conservador

Problemas éticos en la diálisis

- Impacto de las creencias de los profesionales en la toma de decisiones

- Escasos beneficios de dializar a > 75 años

- Valoración de las consecuencias del tratamiento respecto al principio de no maleficencia

- Desproporción terapéutica

Problemas éticos referidos a la información y comunicación

- Pronóstico no discutido de manera habitual

- Temor a ser malinterpretado por iniciar las discusiones de RTRS

- Impacto emocional para todas las partes

- Repercusión en los niveles de burnout de los profesionales

- Falta de formación específica para los profesionales

Problemas éticos en la toma de decisiones y consentimiento

- Dudas éticas sobre si se prolonga la vida del paciente o su muerte

- Desafío a la autonomía del paciente debido a las creencias del profesional

- Toma de decisiones sin la participación del paciente

- Injerencia familiar en la toma de decisiones

- Gestión de recursos limitados

- Emocionalmente parece más difícil retirar un tratamiento que no iniciarlo
Moss, 2000"17; Winearls, 2006 24 ; Cranston, $2008^{25}$; Murtagh, et al., 200910; Rutecki, 2011 ${ }^{19}$;

Schneiderman, 201143; Fassett, et al., 201121;

Brown, 201220; O'Connor, et al., 20124; Lai, et al., 201323; Sánchez-Tomero, 20132; Urban, et al., 201348; Panocchia, et al., 2014 ${ }^{14}$; Schmidt, et al., 2014 ${ }^{26}$; Hussain, et al., 2015'3; Butler, et al., 201638; Findlay, et al., 2016 ${ }^{46}$; Wightman, et al., 2016 ${ }^{15}$; Lazenby, et al., 2017'12;

García-Llana, et al., 201755; Walton, et al., $2017^{18}$ Alonso, 2018

Singer, et al., 199531; Viederman, et al., 197433; Farrington, et al., 201136; Fassett, et al., 201121; Rutecki, 2011 ${ }^{19}$; Leiva-Santos, et al., 201237; 0'Connor, et al., 20124; Lai, et al., 201323; Muthalagappan, et al., 201327; Sánchez-Tomero, 20132; Stewart, et al., 201328; Murphy, et al., 2014'; Noordzij, et al., 201422; Panocchia, et al., 20144, Schmidt, et al., 201426; Balzan, et al., 201529; Hussain, et al., 201513; Butler, et al., 201638; Panocchia, et al., 201711;

Alonso, et al., $2018^{1}$

Singer, et al., 199531; Brown, 2012 ${ }^{20}$; Lai, et al., 201323; Muthalagappan, et al., 201327; Hussain, et al., 2015 ${ }^{13}$; Lim, et al., 201630; Vukusich, et al., 201632; García-Llana, et al., 201755; Lazenby, et al., 2017 ${ }^{12]}$; Pérez-Oliva, et al., 201754; Alonso, et al., 2018'; Axelsson, et al., 2020³

Viederman, et al., 197433; Singer, et al., 199531; Moss, $2000^{17}$; Winearls, 200624; Murtagh, et al., 200910; Farrington, et al., 2011 ${ }^{36}$; Fassett, et al., 2011'; Brown, 2012 ${ }^{20}$; Leiva-Santos, et al., 201237; O'Connor, et al., 20124; Lai, et al., 201323; Muthalagappan, et al., 201327; Sánchez-Tomero, 20132; Stewart, et al., 201328; Aggarwal, et al., 2014 ${ }^{45}$; Feely, et al., 2014 ${ }^{51}$; Schmidt, et al., 201426. Balzan, et al., 2015²9; Hussain, et al., 2015 ${ }^{13}$; van Biesen, et al., 2015 ${ }^{35}$; Butler, et al., 2016 $6^{38}$; Hain, et al., 2016 $6^{44}$; Kahrass, et al., 20165; Lim, et al., 2016 ${ }^{30}$; Vukusich, et al., 201632;

Lazenby, et al., 2017²; Panocchia, et al., 2017"1; Tate, et al., 201750; Alonso, et al., 20181;

Schildmann, et al., 20197; Hernández-Zambrano, et al., 201934; Axelsson, et al., 2020 
Tabla 2. Principales hallazgos de la revisión clasificados según el modelo propuesto por Kahrass, et al., $2016^{5}$ (Continuación)

Hallazgos principales

Artículos

Aspectos sociales y ambientales que influyen en los problemas éticos

- Alto coste económico que condiciona los servicios

- Diferencias regionales en el acceso a los servicios

Winearls, 2006 ${ }^{24}$; Rutecki, 201119; Schneiderman, 201143; Lai, et al., 201323; Schmidt, et al., 201426; Butler, et al., 201638; Moosa, 201641; Salas, 201640; Walton, et al., 201718; Obregón, 201839; da Cunha, $2019^{42}$

Problemas éticos durante el proceso de cuidados y evaluación

- Dificultad para establecer un pronóstico

- Reconocer el momento de la adecuación del esfuerzo terapéutico

- Uso deficitario de recursos paliativos

- Mal control de síntomas asociados

Problemas éticos en grupos con características especiales:

pediatría y demencia

- Diferencias entre opinión paterna y profesional en casos de menores

- Dificultad para determinar cuándo el menor tiene capacidad de decisión

- Conductas disruptivas en pacientes con demencia que dificultan el tratamiento
Viederman, et al., 197433; Moss, 200017; Winearls, 2006 24; Murtagh, et al., 200910; Farrington, et al., 2011 ${ }^{36}$; Fassett, et al., 201121; Rutecki, 2011 ${ }^{19}$; Leiva-Santos, et al., 201237; 0'Connor, et al., 20124; Ishikawa, et al., 201347; Sánchez-Tomero, 20132; Urban, et al., 201348; Aggarwal, et al., 201445; Schmidt, et al., 201426; Panocchia, et al., 201414; Balzan, et al., 201529; van Biesen, et al., 201535; Findlay, et al., 201646; Hain, et al., $2016^{44}$; Kahrass, et al., 20165; Walton, et al., 2017 ${ }^{18}$; Alonso, et al., 2018'; Wetmore, et al., 20189; Hernández-Zambrano, 201934;

Axelsson, et al., $2020^{3}$

Orr, et al., 201052; Feely, et al., 201451; Wightman, et al., 2016 15 ; Tate, et al., 201750; Willig, et al., $2018^{49}$

ERCA: enfermedad renal crónica avanzada; RTRS: retirada de la terapia renal sustitutiva.

diaria, demencia] y comorbilidades asociadas graves) el tratamiento conservador es una alternativa válida que se debe considerar para alcanzar las metas del paciente ${ }^{23}$. Siempre se debe evaluar la capacidad cognitiva y de toma de decisiones de quienes solicitan RTRS para garantizar su comprensión de la magnitud y de las consecuencias de su decisión ${ }^{26,29}$.

\section{Problemas éticos referidos a la información y comunicación}

Los pacientes no plantean la RTRS porque no son conscientes de esta posibilidad, creen que los médicos no la apoyarán, temen ser malinterpretados o no encuentran el lugar adecuado para mantener esas conversaciones ${ }^{1,3,30}$. La ambivalencia de los pacientes también debe ser considerada: se ha visto que los pacientes seguían considerando adecuada su decisión de comenzar la diálisis mientras experimentaban los beneficios del tratamiento, pero una vez que su condición empeoraba cuestionaban esa decisión ${ }^{13,31}$. 
Los profesionales tienen dificultades para transmitir malas noticias e iniciar conversaciones de RTRS, lo cual supone un detrimento de la calidad asistencial. Entre los motivos para ello encontramos el miedo a ser malinterpretados, la falta de consenso sobre quién debe iniciar las conversaciones y la cercanía con los pacientes, que dificulta reconocer y comunicar el deterioro ${ }^{1,3}$. La situación es aún más difícil con pacientes que se sienten bien y con los más jóvenes ${ }^{12}$. Los profesionales reconocen que los problemas éticos a los que más se enfrentan son la desproporción terapéutica y la falta de comunicación entre pacientes, familias y equipo médico ${ }^{32}$ y emplean el término sobrediálisis para referirse a tratamientos prolongados e innecesarios que presencian sin poder evitarlos ${ }^{12}$.

\section{Problemas éticos en la toma de decisiones y consentimiento}

El proceso de toma de decisiones evoluciona junto a la enfermedad: se deben reevaluar los síntomas, la calidad de vida y las relaciones con los demás periódicamente ${ }^{10}$. Se ha evaluado que hasta un $60 \%$ de los pacientes se arrepiente de haber iniciado TRS ${ }^{1,26}$. Generalmente, los pacientes no tienen los recursos emocionales necesarios para hacer frente a las dificultades de la TRS ${ }^{33}$, por lo que son proclives a ceder su autonomía en favor de la opinión de los profesionales ${ }^{13}$. Los pacientes mayores frágiles, en particular, tienen menos tendencia a la RTRS pese a tener peor calidad de vida o pronóstico ${ }^{11}$. La familia es protagonista fundamental cuando se plantea la RTRS y llegar a un acuerdo con ellos puede ser lo más difícil del proceso ${ }^{3}$. A los familiares les cuesta ver una diferencia entre que la muerte llegue sola a que llegue tras participar ellos en una decisión que podía acelerarla. Muchas veces piden que «se haga todo lo humanamente posible»². Esta actitud lleva a conductas "distanásicas», lo que hace que los profesionales eviten y retrasen las discusiones en estos $\operatorname{casos}^{13,23}$ o tiendan a respetar los deseos familiares ${ }^{29}$ por encima de su criterio profesional. Hasta el $\mathbf{8 6 . 8 \%}$ de las decisiones sobre la RTRS puede llegar a tomarlas la familia $^{32}$, aunque esto depende de factores culturales ${ }^{23}$. Las causas del malestar emocional de los pacientes pueden ser múltiples y se ven influidas por su capacidad de afrontamiento, sus ocupaciones, su apoyo social, su capacidad de autocuidado y sus relaciones familiares ${ }^{34}$, así como por la depresión, que es la principal enfermedad psiquiátrica en esta población y la que mayores consecuencias tiene ${ }^{1}$.

Los factores que afectan a los profesionales durante el proceso de toma de decisiones siguen siendo desconocidos ${ }^{13}$. Algunos refieren encontrar emocionalmente más difícil retirar el tratamiento que no iniciarlo ${ }^{21}$. La falta de protocolos específicos puede frenar la actuación de los profesionales, así como su escasa formación en cuidados paliativos ${ }^{35}$. Para los pacientes de ERCA es esencial un programa educativo prediálisis ${ }^{1,36}$, pero muchos profesionales se encuentran con problemas de escasa formación entre sus pacientes ${ }^{3}$, lo que puede comprometer una correcta adhesión al tratamiento ${ }^{36}$. Por todo ello, la decisión del profesional se sustenta más en el criterio médico y en la experiencia que en las preferencias del paciente ${ }^{13}$, contrariamente a lo que debería ocurrir ${ }^{37}$, y es más común que obtengan información de las enfermeras que de los pacientes ${ }^{3}$. Si el paciente es incompetente para tomar la decisión de optar a RTRS, conocer sus ideas previas al respecto influye en los nefrólogos ${ }^{31}$. Por otro lado, Lim et al. ${ }^{30}$ sostienen que plantear estos temas no destruye las esperanzas, ni causa estrés o ansiedad en los pacientes. 


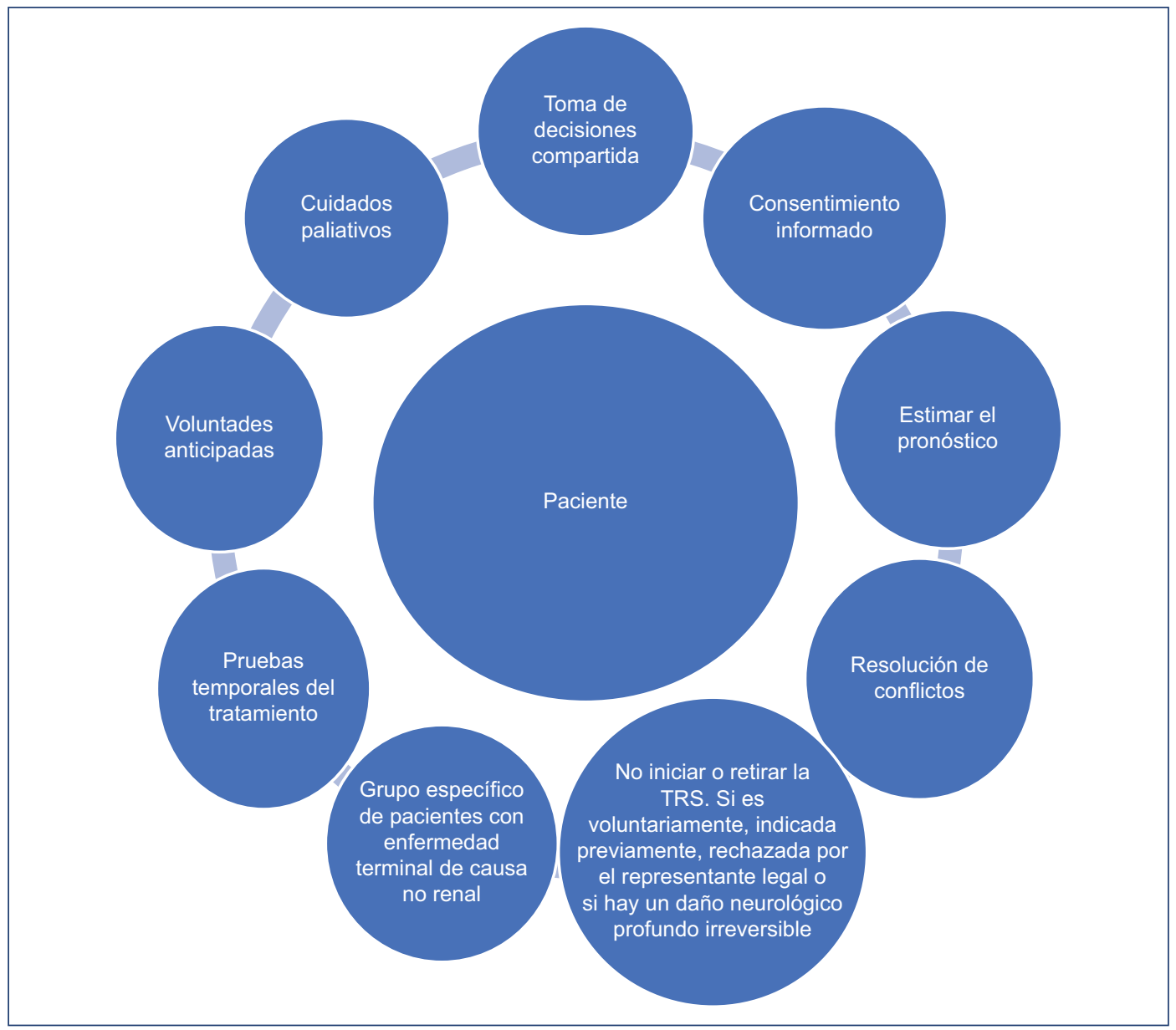

Figura 2. Recomendaciones de la Renal Physicians Association y la American Society of Nephrology para ayudar a la toma de decisiones acerca de iniciar, continuar y parar la terapia renal sustitutiva (TRS) (adaptada de Moss, 200017).

Planificar es fundamental para la RTRS. Para ello puede recurrirse a las voluntades anticipadas (VA), usándolas no solo como la firma de un documento ${ }^{1}$, y un modelo específico de VA para diálisis podría ser mejor aceptado ${ }^{31}$. Se ha puesto de manifiesto la falta de documentos de CPR y VA en pacientes en TRS ${ }^{21}$, lo cual dificulta la toma de decisiones en situaciones de urgencia. La pregunta “¿se sorprendería si este paciente muriese durante el próximo año?" puede ayudar a los profesionales a identificar pacientes susceptibles ${ }^{26}$ de ser abordados para ello. La Renal Physicians Association y la American Society of Nephrology han elaborado una guía de práctica clínica basada en la evidencia para tratar de ayudar a los pacientes, familiares y equipo médico a colaborar para tomar las decisiones acerca de iniciar, continuar y parar la TRS. Sus recomendaciones más destacadas se muestran en la figura 2.

Por otro lado, la toma de decisiones compartida es «la interacción profesional-paciente en la que hay dos vías de intercambio de información con la participación de ambas partes en la 
decisión» . Este modelo busca la atención individualizada y el respeto a la autonomía del paciente que solo será válida si este conoce todas las opciones disponibles ${ }^{38}$. Si tienen elección, los pacientes suelen evitar tratamientos intervencionistas; colateralmente, se consigue un mejor reparto de los recursos y ciertos grupos, como el de pacientes mayores frágiles, se benefician de resultados más personalizados ${ }^{27}$. Las «discusiones éticas caso a caso" son otro método empleado para ayudar en la toma de decisiones que entrañan desafíos éticos. Consisten en pedir la participación de un experto o comité de ética asistencial para ayudar a resolver los conflictos éticos del caso ${ }^{7}$.

\section{Aspectos sociales y ambientales que influyen en los problemas éticos}

Los diferentes tipos de TRS, especialmente la HD, tienen un gran coste económico ${ }^{39}$. Esto tiene repercusiones en los servicios de salud de los países que la subvencionan y en cómo se reparten sus recursos. Por ejemplo, en 2010 el 7.9\% del presupuesto de Medicare (EE.UU) fue para pacientes con ERCA, que son el $1.3 \%$ de los beneficiarios del programa ${ }^{38}$. Algunos cálculos, usando el sistema de años de vida ajustados a la calidad, indican que la diálisis proporciona relativamente poco valor cuando se compara así con otras intervenciones en salud ${ }^{38}$. Esto impone una responsabilidad extra sobre los nefrólogos, pues al admitir pacientes al programa, autorizan un elevado gasto económico ${ }^{23}$. En países donde el modelo de salud no es de capital íntegramente público, los nefrólogos pueden verse influenciados por intereses privados como la ganancia económica, el ahorro, la sobreutilización de recursos y otros desafíos éticos, incluyendo el propio beneficio económico al mantener a alguien en $\mathrm{TRS}^{40}$. En algunos países el debate se centra en el reparto de recursos escasos ${ }^{24}$, situación que lleva a tomar medidas de diversa índole para armonizar los derechos de los individuos y la responsabilidad de la sociedad ${ }^{41}$. En Brasil, Urgencias es la puerta de entrada a TRS y, normalmente, se quedarán hospitalizados para asegurarse el acceso a diálisis hasta tener plaza en una clínica ${ }^{42}$. Los costes médicos deben ser justos y razonables: los recursos siempre son inferiores a las demandas, pues todos los sistemas de salud racionan de una manera u otra. La cuestión es si se distribuyen los recursos de manera transparente y prudente o si se hace de manera implícita en los presupuestos, la burocracia o las carteras de servicios ${ }^{43}$.

\section{Problemas éticos durante el proceso de cuidados y evaluación}

Cuando la beneficencia y la no maleficencia chocan, lo primero es evitar hacer daño ${ }^{44}$. La RTRS se ha convertido en una causa común de muerte para los pacientes en diálisis ${ }^{17,36}$. La media de supervivencia tras RTRS es de 8-12 días ${ }^{2,17}$. Los pacientes que optan a RTRS suelen tener problemas de acceso vascular e inestabilidad hemodinámica postsesión, son más jóvenes, han registrado las VA, han desarrollado comorbilidades y déficits funcionales y son más propensos a elegir morir en su domicilio ${ }^{45}$. Estadísticamente, la RTRS se da más en mujeres mayores con historia de accidente vascular cerebral y hospitalizaciones previas largas ${ }^{46}$ y pocos pacientes deciden RTRS sin encontrarse en un episodio agudo o de rápido deterioro ${ }^{45}$. Toda RTRS debe incluir cuidados paliativos $2,18,21,36,46$ : no hacerlo puede incrementar el sufrimiento del paciente y de los cuidadores $^{34,46}$. El objetivo del manejo clínico conservador es retrasar la progresión de la enfermedad y tratar las complicaciones derivadas ${ }^{1,4}$ para ayudar al paciente a vivir y morir de manera confortable sin TRS ${ }^{24}$. Hasta un $60 \%$ de los pacientes de diálisis sufren infratratamiento del dolor 
y otros síntomas que afectan a su calidad de vida ${ }^{1,4,46}$, posiblemente por una incorrecta evaluación del dolor ${ }^{1}$ y por el escaso uso de opioides, pese a estar recomendados ${ }^{47}$. Resulta paradójico que estos pacientes reciban tratamientos tan sofisticados, pero carezcan de otros básicos como el control de síntomas o cuidados paliativos ${ }^{10}$.

Todos los pacientes tienen el mismo derecho a acceder a cuidados paliativos de calidad, siempre respetando su autonomía ${ }^{45}$. Los nefrólogos tienen el deber de iniciar discusiones sobre el fin de la vida con los pacientes que lo requieran ${ }^{26}$. Existe una asociación significativa entre incremento en número de RTRS y formación específica en paliativos ${ }^{35}$, la cual, además, va en aumento ${ }^{19}$. Asimismo, la colaboración entre unidades supone una mejoría en la calidad percibida por los familiares de los pacientes ${ }^{48}$. La guía sobre cuidados de soporte paliativo en el paciente renal ${ }^{1}$ y el Plan de Cuidados Paliativos Renales Avanzados son herramientas para el manejo de los síntomas, para la implementación de medidas de confort y para desarrollar habilidades de comunicación.

\section{Problemas éticos en grupos con características especiales: pediatría y demencia}

En pediatría, la decisión de no empezar el tratamiento es más común que la de retirarlo ${ }^{15}$. Se ha defendido, apelando al principio de no maleficencia, que cuando un niño no es candidato a trasplante, la diálisis puede ser un instrumento que prolongue el proceso de la muerte por lo que sería lícito no iniciarla. No obstante, a los adultos se les ofrece la diálisis sean o no candidatos a trasplante, por lo que se puede hablar de vulneración del principio de justicia a los niños al no ofrecerles las mismas opciones que a los adultos ${ }^{15}$. La decisión, en todo caso, siempre debe garantizar el bien superior del menor, por lo que se debe buscar un consenso entre padres y profesionales, evitando la judicialización del caso ${ }^{15,49}$. Otro aspecto ético importante es determinar cuándo un adolescente puede ser considerado maduro para tomar decisiones de RTRS ${ }^{50}$.

Por otro lado, están los pacientes con conductas que, sea por enfermedad, problemas psicológicos $u$ otros, pueden poner en riesgo a sí mismos, a otros usuarios o al personal del servicio y que, tras diversas intervenciones, no consiguen ser controladas. La seguridad y responsabilidad ética hacia los otros pacientes y el personal podrían justificar la RTRS del paciente ${ }^{51}$, aunque previamente se pueden negociar opciones y límites tolerables o intentar el traslado a otro centro ${ }^{52}$.

\section{Discusión}

En nuestro análisis hemos seguido el modelo propuesto por Kahrass, et al. ${ }^{5}$ para homogeneizar la evidencia y el consecuente debate bioético. Aunque el objetivo de este artículo no es analizar los aspectos éticos de la inclusión de pacientes en TRS, es importante tener en cuenta ciertos problemas éticos que se dan al diagnosticar la ERC. Las características epidemiológicas de la población susceptible de TRS nos llevan a nuevos retos éticos ligados a la edad de los pacientes, su capacidad para consentir (sobre todo cuando las decisiones se retrasan a momentos de agudizaciones) y la idoneidad de un tratamiento con poco margen de ventajas-inconvenientes. No existen datos concluyentes acerca de la idoneidad de la TRS sobre el tratamiento conservador 
para ciertos grupos etarios. Esto hace imprescindible que las decisiones se tomen de manera compartida e individualizada para cada caso. La comunicación se ve afectada por todo esto, lo que lleva a creencias erróneas y a retrasar decisiones importantes como plantear desde el inicio la necesidad en algún momento de un plan de $\mathrm{CPR}^{3,6,7,12,13,21}$.

Las decisiones en salud que toman los pacientes no son permanentes, al igual que no lo es el propio proceso de salud-enfermedad, por eso es pertinente hacer una reevaluación periódica, siempre respetando su autonomía. La influencia de las familias suele decantar la balanza hacia el respeto de sus propios deseos con independencia de los del paciente ${ }^{13,23,29,32}$. Existen numerosos datos acerca de la esperanza de vida y de los efectos en la calidad de vida de los pacientes en TRS que llevan al profesional a considerar la adecuación del tratamiento. La RTRS es una forma de $\mathrm{AET}^{1,9} \mathrm{y}$, como tal, queda amparada por la legalidad en nuestro país. A ello hay que añadir que es un procedimiento que forma parte de la buena práctica médica diaria, es éticamente correcto y aceptable, justificado dentro del marco de la bioética y que, como tal, es una obligación moral de los profesionales considerarlo cuando sea pertinente ${ }^{53}$. A pesar de ello, nuestro análisis muestra numerosos problemas para iniciar estas conversaciones. El proceso de transmisión de información se ha estudiado principalmente con encuestas que muestran el temor de pacientes y profesionales a iniciar las conversaciones acerca de la RTRS y una evidente falta de formación en comunicación de los profesionales $1,27,54$. Las habilidades comunicativas son una herramienta esencial para el profesional de la salud que debe seguirse desarrollando de forma continuada ${ }^{55}$.

Por otro lado, Panocchia, et al. ${ }^{11}$ llaman la atención sobre la negativa de numerosos pacientes a participar en su estudio por tratarse en él temas estresantes. Otros estudios no especifican esta problemática, por lo que sería prudente considerar hasta qué punto se infravalora el daño potencial que se puede causar a los participantes al abordar estas materias y si son representativos los resultados al no haber participado un grupo de pacientes concreto. Richards, et al. ${ }^{56}$ analizan cuatro riesgos potenciales para los participantes de la investigación cualitativa: ansiedad y angustia, explotación, tergiversación e identificación del participante en trabajos publicados. Como estrategias para reducir el riesgo de daños, recomiendan garantizar la solidez científica, organizar la atención de seguimiento cuando sea apropiado, obtener el consentimiento, garantizar la confidencialidad y adoptar una postura reflexiva frente al análisis. No iniciar conversaciones de RTRS en el momento adecuado tiene consecuencias para los pacientes: si no son proactivas, suelen tener lugar cuando el paciente ya no puede participar ${ }^{3}$. Así, el $50-80 \%$ de los pacientes llegan al momento de la RTRS siendo incompetentes para la toma de decisiones ${ }^{48}$. Esto puede evitarse con una buena planificación anticipada de los cuidados que permita respetar su autonomía. En todo momento, el profesional debe atender a su deber de no maleficencia y evitar un tratamiento fútil o perjudicial para el paciente. Un caso muy estresante para los profesionales es cuando pacientes con buena calidad de vida piden RTRS, ya que choca con las creencias del profesional. Se recomienda que se compruebe la capacidad del paciente de tomar estas decisiones para garantizar un adecuado proceso de RTRS acorde a la legislación y al principio bioético de autonomía.

Los problemas comunicativos afectan a los profesionales y se reflejan en los índices de burnout. Algunos de los estresores más comunes son las relaciones con pacientes y familiares, las cargas de trabajo, el contacto directo con la enfermedad y el dolor, el ofrecer asistencia de calidad y el equilibrio entre beneficencia, no maleficencia y autonomía ${ }^{1,3}$. La mayoría de los profesionales sanitarios son testigos habituales de la fragilidad, el sufrimiento, el dolor y la muerte en su trabajo, lo que aumenta 
el riesgo de burnout, fatiga por compasión, problemas morales y vulnerabilidad. Esto ocurre especialmente si carecen de conciencia de sí mismos y de un entorno de trabajo adecuado ${ }^{57}$, por lo que es necesario que desarrollen una conciencia crítica de su situación ${ }^{39}$. En España, el índice del 11-23\% de burnout en personal sanitario general aumenta a un 27-39\% en nefrología ${ }^{1}$. La comunicación también es un factor clave para la toma de decisiones y el consentimiento, sobre todo cuando el paciente no puede consentir y no ha dejado indicaciones expresas. En estos casos, la familia debe actuar como garante de la autonomía del paciente. Es necesario capacitar a pacientes y familiares para la toma de decisiones, ya que el proceso dependerá no solo de quién toma las decisiones, sino de cómo se toman. Se necesitan más guías e instrumentos que ayuden en este proceso $0^{1,13}$. El profesional debe evitar un excesivo recelo por la vulnerabilidad de los pacientes para prevenir caer inintencionadamente en una postura de «paternalismo selectivo»1. Vulnerar la autonomía del paciente puede suponer dañarlo; solo conociendo los verdaderos deseos del paciente se puede establecer un curso correcto de actuación ${ }^{3}$. Las malas noticias solo deberían ocultarse si la persona así lo ha requerido 0 ante el riesgo de un grave impacto negativo en el paciente ${ }^{44}$. Se recomienda que la decisión sobre AET se base en la valoración del pronóstico y trayectoria esperada, la detección de síntomas refractarios, la presencia de criterios de enfermedad terminal y, sobre todo, los deseos del paciente ${ }^{1}$.

Existen aspectos sociales y ambientales que comprometen el principio de justicia. Se ha investigado poco acerca de cómo la disponibilidad de recursos afecta a los criterios nefrológicos para el reparto de recursos ${ }^{10}$ y de inclusión en TRS ${ }^{41}$. En países como Sudáfrica los pacientes son de media 10 años más jóvenes, se da preferencia a los que tienen trabajo, esposa e hijos y a los no diabéticos ${ }^{41}$, criterios similares a los usados por el primer comité de bioética en Seattle en la década de $1960^{16}$. A mayor financiación, más pacientes admitidos, mayor número de ellos frágiles, y más casos de RTRS ${ }^{35}$. La cultura y la legislación también afectan a la RTRS. En países como Japón solo cuando el paciente es considerado terminal puede procederse a la RTRS ${ }^{58}$, lo que dificulta el debate ${ }^{47}$ sobre el tema. Los cuidados paliativos son indispensables tras la RTRS y parece haber consenso sobre la necesidad de integrarlos en los cuidados de la TRS ${ }^{1,10}$. No obstante, al no hablar de RTRS o de la inminencia de la muerte tampoco se habla de CPR, por lo que prevalece un mal control de síntomas terminales en pacientes que aún reciben diálisis $1,10,46$.

Por último, en situaciones especiales, como en pediatría, la toma de decisiones debe ser llevada a cabo conjuntamente por el equipo sanitario y los padres, teniendo en cuenta la opinión del niño (tras las modificaciones que la Ley 25/2015, de 28 de julio, introdujo a la Ley 41/2002, de 14 de noviembre), con el objetivo de velar por el interés superior del menor ${ }^{1}$. En cuanto a la demencia, esta es la única variable que se asocia significativamente a RTRS ${ }^{48}$ y hasta en un $80 \%$ de esos casos se pide una valoración bioética ${ }^{10}$. En una sociedad que prioriza la autonomía, tomar decisiones para pacientes no representados es irremediablemente un proceso éticamente complejo. Por ello, se deben identificar a tiempo los casos de riesgo, documentar sus deseos y buscarles representantes llegado el caso $^{59}$.

\section{Limitaciones del estudio}

Nuestro estudio presenta algunas limitaciones. Primero, la falta de consenso académico acerca de los términos que se deberían emplear como palabras clave y la gran dispersión del campo nos llevaron a realizar tres búsquedas independientes variando los términos diálisis, hemodiálisis y 
diálisis renal, con el consecuente riesgo de no haberse localizado artículos que usan terminología diferente. En segundo lugar, la limitación del idioma a español e inglés puede haber excluido trabajos relevantes en otros idiomas. En tercer lugar, el uso de la clasificación de Kahrass, et al. ${ }^{5}$ puede haber influido en la importancia dada a los diferentes dilemas éticos, pudiendo parecer de mayor relevancia los apartados que concentran más resultados sin que esto refleje la realidad.

\section{Conclusión}

La RTRS, como forma de AET, es ética y legalmente aceptable en pacientes con comorbilidades, problemas sintomáticos y corta esperanza de vida. Se debe realizar con unas medidas adecuadas de confort y acompañarse de soporte paliativo para evitar el mal control de síntomas que se evidencia en la literatura. La toma de decisiones compartida es un instrumento que ayuda a minimizar la vulneración del principio de autonomía, ya que posibilita una planificación anticipada de cuidados. Existe una fuerte demanda de formación específica en comunicación por parte de los profesionales. Para un adecuado reparto de los recursos sanitarios se deben equilibrar los valores individuales y colectivos. Esta gestión de recursos puede influenciar los criterios de admisión en diálisis, por lo que es necesaria una valoración ética. Se necesitan guías que ayuden en el proceso de toma de decisiones, así como más formación en bioética.

\section{Conceptos clave}

- La RTRS implica grandes desafíos éticos.

- Es necesario sistematizar las recomendaciones éticas disponibles respecto a la RTRS.

- Debe realizarse con asistencia de calidad y acompañarse de cuidados paliativos.

- Se necesita formación de los profesionales en habilidades comunicativas y bioética.

\section{Financiamiento}

La presente investigación no ha recibido ninguna beca específica de agencias de los sectores público, comercial o sin ánimo de lucro.

\section{Conflicto de intereses}

Los autores declaran no tener conflicto de intereses.

\section{Responsabilidades éticas}

Protección de personas y animales. Los autores declaran que para esta investigación no se han realizado experimentos en seres humanos ni en animales. 
Confidencialidad de los datos. Los autores declaran que en este artículo no aparecen datos de pacientes.

Derecho a la privacidad y consentimiento informado. Los autores declaran que en este artículo no aparecen datos de pacientes.

\section{Bibliografía}

1. Alonso A, García H, Leiva JP, Sánchez R (coordinadores). Cuidados paliativos en enfermedad renal crónica avanzada. SEN-SECPAL. Madrid: Pulso Ediciones; 2018.

2. Sánchez-Tomero JA. Reflexiones sobre la entrada y la retirada de diálisis. Nefrologia. 2013;33(6):758-3.

3. Axelsson L, Benzein E, Lindberg J, Persson C. Processes toward the end of life and dialysis withdrawal physicians and nurses perspectives. Nurs Ethics. 2020;27(2):419-32.

4. O'Connor NR, Kumar P. Conservative management of end-stage renal disease without dialysis: A systematic review. J Palliat Med. 2012;15(2):228-5.

5. Kahrass $\mathrm{H}$, Strech $\mathrm{D}$, Mertz $\mathrm{M}$. The full spectrum of clinical ethical issues in kidney failure. Findings of a systematic qualitative review. PLoS One. 2016;11(3):1-17.

6. Qazi HA, Chen H, Zhu M. Factors influencing dialysis withdrawal: A scoping review. BMC Nephrology. 2018;19(96):1-11.

7. Schildmann J, Nadolny S, Haltaufderheide J, Gysels M, Vollmann J, Bausewein C. Ethical case interventions for adult patients. Cochrane Database Syst Rev. 2019 Jul 22;7(7):CD012636. doi: 10.1002/14651858.CD012636.pub2. Online ahead of print.

8. Murphy E, Germain MJ, Cairns H, Higginson IJ, Murtagh FEM. International variation in classification of dialysis withdrawal: a systematic review. Nephrol Dial Transplant. 2014;29:625-35.

9. Wetmore JB, Yan H, Hu Y, Gilbertson DT, Liu J. Factors associated with withdrawal from maintenance dialysis: A case-control analysis. Am J Kidney Dis. 2018;71(6):831-41.

10. Murtagh FEM, Spagnolo AG, Panocchia N, Gambaro G. Conservative (non dialytic) management of end-stage renal disease and withdrawal of dialysis. Prog Palliat Care . 2009:17(4):179-4.

11. Panocchia N, Tonnara G, Minacori R, Sacchini D, Bossola M, Tazza L, et al. Survey on advance care planning of Italian outpatients on chronic haemodialysis. BMJ Support Palliat Care. 2017;7:419-2.

12. Lazenby S, Edwards A, Samuriwo R, Riley S, Murray MA, Carson-Stevens A. End-of-life care decisions for haemodialysis patients - 'We only tend to have that discussion with them when they start deteriorating'. Health Expect. 2016;20:260-73.

13. Hussain JA, Flemming K, Murtagh FEM, Johnson MJ. Patient and health care professional decision-making to commence and withdraw from renal dialysis: A systematic review of qualitative research. Clin J Am Soc Nephrol. 2015;10:1201-15.

14. Panocchia N, Bossola M, Tazza L. Decision-making in the implementation or withdrawal of dialysis in the old complex patient. Italian Journal of Medicine. 2014;8:6-10.

15. Wightman AG, Freeman MA. Update on ethical issues in paediatric dialysis: Has paediatric dialysis become morally obligatory? Clin J Am Soc Nephrol. 2016;11:1456-2.

16. Aulisio MP. Why did hospital ethics committees emerge in the US? AMA J Ethics. 2016;18(5):546-3.

17. Moss $\mathrm{AH}$. A new clinical practices guideline on initiation and withdrawal of dialysis that makes explicit the role of palliative medicine. $J$ Palliat Med. 2000;3(3):253-0.

18. Walton LS, Shumer GD, Thorsteinsdottir B, Suh T, Swetz KM. Palliation versus dialysis for end-stage renal disease in the oldest old: What are the considerations? Palliat Care. 2017;10:1-5.

19. Rutecki GW. Would treatment allocation according to age-contingent depreciation be ethical? A dialysis \& transplantation paradigm. Ethics \& Medicine. 2011;27(2):99-7.

20. Brown EA. Non-dialysis therapy: A better policy than dialysis followed by withdrawal? Semin Dial. 2012;25(1):26-7.

21. Fassett RG, Robertson IK, Mace R, Youl L, Challenor S, Bull R. Palliative care in end-stage kidney disease. Nephrology. 2011;16:4-12.

22. Noordzij M, Jager KJ. Increased mortality early after dialysis initiation: a universal phenomenon. Kidney Int. 2013;85:12-4.

23. Lai CF, Tsai HB, Hsu SH, Chiang CK, Huang JW, Huang SJ. Withdrawal from long-term haemodialysis in patients with end-stage renal disease in Taiwan. J Formosan Med Association. 2013;112:589-9.

24. Winearls CG. In the wake of progress - Ethical problems of renal failure treated by dialysis. Clin Med. 2006;6(1):76-80.

25. Cranston R. Clinical ethics dilemma: Is it permissible to forgo life-saving dialysis? Ethics \& Medicine. 2008;24(2):83-5.

26. Schmidt RJ, Moss AH. Dying on dialysis: The case for a dignified withdrawal. Clin J Am Soc Nephrol. 2014;9:174-0.

27. Muthalagappan S, Johansson L, Kong WM, Brown EA. Dialysis or conservative care for frail older patients: Ethics of shared decision-making. Nephrol Dial Transplant. 2013:28:2717-2.

28. Stewart C, Brennan F. Legal issues concerning withholding and withdrawal of dialysis. Nephrology. 2013;18:444-1.

29. Balzan JM, Cartier JC, Calvino-Gunther S, Carron PL, Baro P, Palacin P, et al. Dialysis withdrawal: Impact and evaluation of a multidisciplinary deliberation within an ethics committee as a Shared-Decision-Making Model. Ther Apher Dial. 2015;19(4):385-2.

30. Lim CED, Ng RWC, Cheng NCL, Cigolini M, Kwok C, Brennan F. Advance care planning for haemodialysis patients. Cochrane Database Syst Rev. 2016;7(7):CD010737.

31. Singer PA, Thiel EC, Naylor CD, Richardson RMA, Llewellyn-Thomas H, Goldstein M, et al. Life-sustaining treatment preferences of haemodialysis patients: Implications for advance directives. J Am Soc Nephrol. 1995;6(5):1410-7.

32. Vukusich A, Catoni MI, Salas SP, Valdivieso A, Browne F, Roessler E. Problemas ético-clínicos en hemodiálisis crónica: percepción de médicos y enfermeras. Rev Med Chile. 2016;144:14-21.

33. Viederman M, Burke D. Case studies in bioethics: Saying "no" to hemodialysis. Should a minor's decision be respected? Hastings Cent Rep. 1974;4(4):8-10.

34. Hernández-Zambrano SM, Torres-Melo ML, Barrero-Tello SS, Saldaña-García IJ, Sotelo-Rozo KD, Carrillo-Algarra AJ, et al. Needs of palliative care in haemodialysis perceived by patients, principal caregivers and nursing professionals. Enferm Nefrol. 2019;22(2):141-9.

35. van Biesen W, van de Luijtgaarden MWM, Brown EA, Michel JP, van Munster BC, Jager KJ, et al. Nephrologists' perceptions regarding dialysis withdrawal and palliative care in Europe: Lessons from a European Renal Best Practice survey. Nephrol Dial Transplant. 2015;30:1951-8. 
36. Farrington K, Warwick G. Renal Association Clinical Practice Guideline on planning, initiating and withdrawal of renal replacement therapy. Nephron Clin Pract. 2011;118(s1):189-208.

37. Leiva-Santos JP, Sánchez-Hernández R, García-Llana H, Fernández-Reyes MJ, Heras-Benito M, Molina-Ordas Á, et al. Cuidados de soporte renal y cuidados paliativos renales: revisión y propuesta en terapia renal sustitutiva. Nefrologia. 2012;32(1):20-7.

38. Butler CR, Mehrotra R, Tonelli MR, Lam DY. The evolving ethics of dialysis in the United States: a principlist bioethics approach. Clin J Am Soc Nephrol. 2016;11:704-9.

39. Obregón JM, Anjos MF. Nephrologists between power and vulnerability in times of technology. Braz J Nephrol. 2018;40(4):403-9.

40. Salas SP, Vukusich A, Catoni MI, Valdivieso A, Roessler E. Conflictos de intereses en nefrología. Rev Med Chile. 2016;144:1053-8.

41. Moosa MR, Maree JD, Chirehwa MT, Benatar SR. Use of the 'Accountability for reasonableness' approach to improve fairness in accessing dialysis in a middle-income country. PLoS One. 2016;11(10):1-16.

42. da Cunha R, Silva E. Dialysis and the right to die. Rev Bioet. 2019;27(3):394-400.

43. Schneiderman LJ. Rationing just medical care. Am J Bioethics. 2011;11(7):7-4

44. Hain DJ, Diaz D, Paixao R. What are ethical issues when honoring an older adult's decision to withdraw from dialysis. Nephrol Nurs J. 2016;43(5):429-4.

45. Aggarwal Y, Baharani J. End-of-life decision making: withdrawing from dialysis: a 12-year retrospective single centre experience from the UK. BMJ Support Palliat Care. 2014; 4:368-6.

46. Findlay MD, Donaldson K, Doyle A, Fox JG, Khan I, McDonald J, et al. Factors influencing withdrawal from dialysis: a national registry study. Nephrol Dial Transplant. 2016;31:2041-8.

47. Ishikawa H, Ogihara N, Tsukushi S, Sakamoto J. Withdrawal from dialysis and palliative care for severely ill dialysis patients in terms of patient-centred medicine. Case Rep Nephrol. 2013;2013:761691.

48. Urban AK, Brennan F. Patients who withdraw from dialysis in a Sydney centre with palliative care support: who, why, and how do our patients die? Prog Palliat Care. 2013;21(6):325-0.

49. Willig L, Paquette E, Hester DM, Warady BA, Lantos JD. Parents refusing dialysis for a 3-month-old with renal failure. Pediatrics. 2018;141(3):1-6.

50. Tate T, Goldberg A, Wightman A, Warady BA, Lantos JD. Controversy about dialysis for an adolescent. Pediatrics. 2017;140(1):1-6.

51. Feely MA, Albright RC, Thorsteinsdottir B, Moss AH, Swetz KM. Ethical challenges with haemodialysis patients who lack decision-making capacity: behavioural issues, surrogate decision-makers, and end-of-life situations. Kidney Int. 2014;86:475-0.

52. Orr RD, Rutecki GW. Clinical ethics dilemmas: To dialyze or not to dialyze. Ethics \& Medicine. 2010;26(1):11-4.

53. Betancourt RGL, Betancourt BGJ. Adecuación del esfuerzo terapéutico: aspectos éticos y legales relacionados con su práctica. Rev Cub Med Int Emerg. 2017;16(1):12-4.

54. Pérez-Oliva JF, Cabrera MB, Llantá MC, Monzón ME. Necesidades de aprendizaje en bioética de residentes y especialistas de nefrología en Cuba. Rev Habanera de Ciencias Médicas. 2016;16(1):122-34.

55. García-Llana H, Bajo MA, Barbero J, Selgas R, del Peso G. The Communication and Bioethical Training (CoBiT) Program for assisting dialysis decision-making in Spanish ACKD units. Psychol Health Med. 2017;22(4):474-2.

56. Richards H, Schwartz L. Ethics of qualitative research: Are there special issues for health services research? Fam Pract. 2002;19:135-9.

57. Delgado J, de Groot J, McCaffrey G, Dimitropoulos G, Sitter KC, Austin W. Communities of practice: acknowledging vulnerability to improve resilience in healthcare teams. J Med Ethics. 2020 Jan 24;medethics-2019-105865. doi: 10.1136/medethics-2019-105865. Online ahead of print.

58. Tanaka M, Kodama S. Ethical issues around the withdrawal of dialysis treatment in Japan. Asian Bioeth Rev. 2020;12(1):51-7.

59. Hulkower A, Garijo-Garde S, Flicker LS. Should dialysis be stopped for an unrepresented patient with metastatic cancer? AMA J Ethics. 2019;21(7):E575-1. 